\title{
Periodic selection in longterm continuous-flow cultures of the filamentous fungus Fusarium graminearum
}

\author{
Marilyn G. Wiebe, ${ }^{1 *}$ Geoffrey D. Robson, ${ }^{1}$ Bryan Cunliffe, ${ }^{1}$ Stephen G. Oliver ${ }^{2}$ \\ and Anthony P. J. TRINCI ${ }^{1}$ \\ ${ }^{1}$ Microbiology Group, Department of Cell and Structural Biology, School of Biological Sciences, University of \\ Manchester, Manchester M13 9PT, UK \\ ${ }^{2}$ Manchester Biotechnology Centre, University of Manchester Institute of Science and Technology, PO Box 88, \\ Manchester M60 1QD, UK
}

(Received 29 March 1993; revised 9 June 1993; accepted 15 June 1993)

\begin{abstract}
By monitoring increases and decreases in the proportion of cycloheximide-resistant macroconidia, periodic selection was observed in populations of the filamentous fungus Fusarium graminearum, grown in glucose-limited chemostat cultures. The results indicated that periodic selection of advantageous mutants of $F$. graminearum occurred at intervals of about $124 \mathrm{~h}$ at both high $\left(D=0.19 \mathrm{~h}^{-1}\right.$, approximately 34 generations) and low $\left(D=0.06 \mathrm{~h}^{-1}\right.$, approximately 11 generations) dilution rates. Several 'adaptive' peaks (each indicating the appearance of an advantageous mutation) were observed before morphological (highly branched) mutants appeared in the populations; these mutants have previously been observed to have a selective advantage over the parental strain. At intervals, macroconidia harvested from the chemostat were used to inoculate plates of non-antibioticcontaining agar medium, and it was possible to monitor periodic selection in the original chemostat culture using second generation macroconidia harvested from these cultures. The proportion of cycloheximide-, potassium chlorate-, and p-fluoro-DL-phenylalanine-resistant macroconidia in these second generation macroconidia changed in a pattern similar to that observed when monitoring the proportion of cycloheximide-resistant macroconidia in the first generation population harvested directly from the chemostat. The experiments demonstrated that populations of filamentous fungi are heterogeneous and that much of this heterogeneity may already be present at the end of batch growth, i.e. before the onset of continuous cultivation.
\end{abstract}

\section{Introduction}

Although neutral mutations accumulate at a linear rate in continuous cultures (Novick \& Szilard, 1950), their concentration periodically decreases; this periodic removal of neutral mutations from a chemostat population has been termed periodic selection (Dykhuizen \& Hartl, 1983). These periodic events occur when a mutant arises which has a selective advantage over the rest of the population but does not carry the neutral mutation being monitored. As the mutant with the selective advantage replaces the original population, neutral mutations accumulate in the population of the new strain. Periodic selection has been observed in bacterial populations (Helling et al., 1987) and in both haploid and diploid populations of Saccharomyces cerevisiae (Paquin \&

*Author for correspondence. Tel. +44612753918 ; fax +4461275 5656.

Abbreviation: FPA, $p$-fluoro-DL-phenylalanine.
Adams, 1983a), and the implications of these observations for prokaryote and eukaryote evolution have been discussed (Koch, 1974; Charlesworth, 1983; Paquin \& Adams, $1983 a, b)$.

Wiebe et al. (1991) have described the appearance of morphological (highly branched) mutants (known as 'colonial' mutants) in continuous-flow cultures of the filamentous fungus Fusarium graminearum. These mutants have a strong selective advantage over the original population in the cultural conditions in which they arise and could represent the appearance in the population of the first mutant with a selective advantage, which would then be expected to evolve further. Alternatively, other mutants with selective advantages may have arisen in the population prior to the appearance of the morphological mutants, but their phenotype may not have been recognized.

Periodic selection has not previously been observed in populations of filamentous fungi, which being coenocytic (and sometimes heterokaryotic) differ considerably from 
unicellular bacteria and yeasts. Cycloheximide resistance was the marker chosen to monitor periodic selection in $F$. graminearum, as resistance to this antibiotic has been used to monitor adaptive changes in chemostat populations of haploid and diploid populations of Saccharomyces cerevisiae (Paquin \& Adams, 1983a).

Because it is a dominant marker (Hsu, 1963; Paquin \& Adams, 1983a), the frequency of cycloheximide resistant nuclei in a population of coenocytic mycelia could be over-estimated. Consequently, periodic selection in a population of $F$. graminearum is most efficiently monitored by looking for antibiotic resistance in macroconidia, as sporulation represents a way of isolating individual nuclei from the mycelia from which they are formed. $F$. graminearum produces macroconidia in glucose-limited chemostat culture, even at high dilution rates (Wiebe \& Trinci, 1991); only multinucleate macroconidia are produced by this species, but the nuclei in one macroconidium all derive (by mitosis) from a single nucleus in the phialide (Miller, 1946). Thus, macroconidia are multinucleate, but homokaryotic. Assuming that all nuclei in a coenocytic mycelium have an equal probability of being incorporated into a macroconidium, problems in interpretation of the results, which would arise in monitoring periodic selection using fragments of heterokaryotic mycelia, can be avoided. Consequently, it was possible to carry out experiments, in which the proportion of neutral mutations in the population could be monitored by sampling macroconidia. This paper describes the occurrence of periodic selection events in chemostat-grown populations of $F$. graminearum and discusses the significance of these events.

\section{Methods}

Organism and media. Fusarium graminearum Schwabe strain A3/5 was obtained from Mr T. W. Naylor, Marlow Foods, Billingham, UK. Stock cultures were maintained as macroconidia at $-70^{\circ} \mathrm{C}$ in $20 \%$ (v/v) glycerol. Inocula were prepared as described previously (Wiebe $t$ al., 1991).

The defined medium of Vogel (1956) was used with glucose as the carbon source instead of sucrose. In submerged cultures, $\left(\mathrm{NH}_{4}\right)_{2} \mathrm{SO}_{4}$ was substituted for $\mathrm{NH}_{4} \mathrm{NO}_{3}$ as the nitrogen source. Vogel's mineral salts solution was prepared at $50 \times$ final concentration, sterilized by membrane filtration $(0.2 \mu \mathrm{m}$ pore diam.) and added to the sterile glucose solution. Glucose solutions [final concn $3.0 \mathrm{~g}$ glucose $(1 \text { medium })^{-1}$ ] for continuous-flow cultures were prepared in 10 litre volumes and autoclaved for $60 \mathrm{~min}$ at $121^{\circ} \mathrm{C}$. No caramelization and no significant loss of glucose was observed. For plate cultures, Vogel's modified medium containing $10 \mathrm{~g}$ glucose $\mathrm{l}^{-1}$ was solidified with agar (Davis Gelatine; $15 \mathrm{~g}^{-1}$, final concn).

For media to detect resistant strains, cycloheximide $(0.25$ or $0.75 \mathrm{~mm}$, final concn), potassium chlorate ( $300 \mathrm{mM}$, final concn) or $p$-fluoro-DLphenylalanine (FPA, $5.5 \mathrm{~mm}$, final concn) was added to modified Vogel's medium.

Chemostat cultures. Cultures were grown in a Braun Biostat $\mathbf{M}$ (2 litre) fermenter at $25^{\circ} \mathrm{C}$ as described by Wiebe \& Trinci (1991).
Biomass retention in the fermenter vessel was monitored by taking dry weight measurements of culture samples both from inside the fermenter vessel and from the overflow. No appreciable retention of biomass in the vessel was observed.

Monitoring of cycloheximide resistance and morphological (' colonial') mutations. Samples were removed daily from the fermenter vessel. Macroconidia were separated from the mycelial biomass by filtering the culture suspension through two layers of sterile lens tissues. Counts of viable macroconidia were made on modified Vogel's medium solidified with agar (five or ten replicates per sample), as described by Wiebe $e t$ al. (1991). Approximately $3 \times 10^{4}$ macroconidia per plate were also inoculated onto Vogel's medium containing $0.25 \mathrm{~mm}$-cycloheximide (five or ten replicates per sample). The plates were incubated at $25^{\circ} \mathrm{C}$ for $3 \mathrm{~d}$ (for viable counts) or 6-8 d (to detect cycloheximide resistance). In filamentous fungi, the time (lag phase) between inoculation and germination (and, hence, colony expansion) is altered by cycloheximide (Hsu, 1963; Trinci \& Gull, 1970; Pongratz \& Klingmüller, 1973) and therefore the incubation period was extended appropriately.

Highly branched, morphological mutants were identified from their 'colonial' morphologies and the proportion of 'colonial' mutants to parentals was determined in the total culture population (i.e. macroconidia and mycelial fragments) as described by Wiebe et al. (1991).

Analysis of periodic selection from second generation macroconidia. Macroconidia taken directly from the fermenter vessel were considered to be first-generation macroconidia. For each fermenter sample, about $3 \times 10^{4}$ first-generation macroconidia were inoculated onto plates of non-antibiotic-containing modified Vogel's medium solidified with agar and incubated for at least $8 \mathrm{~d}$ at $25^{\circ} \mathrm{C}$. Macroconidia harvested (in sterile distilled water) from these plates were designated secondgeneration macroconidia. Suspensions of second-generation macroconidia were diluted for viable counts. In one experiment, antibiotic resistance in the second-generation macroconidial population was analysed on two concentrations of cycloheximide (0.25 and $0.75 \mathrm{mM})$, and in a second experiment, resistance of second-generation macroconidia to chlorate and FPA was analysed. Plates were incubated at $25^{\circ} \mathrm{C}$ for 7 and $8 \mathrm{~d}$, respectively, for the two experiments.

\section{Results}

\section{The use of cycloheximide resistance as a marker for a} neutral mutation

$F$. graminearum was sensitive to cycloheximide, but with spontaneous resistance (to $0.25 \mathrm{~mm}$-cycloheximide) occurring in $0 \cdot 001-0.1 \%$ of the population. Cycloheximide resistance was an easy marker to monitor and adaptive peaks were readily recognized. However, a $24-48 \mathrm{~h}$ lag in the germination of cycloheximide-resistant macroconidia of $F$. graminearum was observed in the presence of cycloheximide.

In one experiment, the number of cycloheximideresistant colonies was counted on days 4-8 after inoculation (Fig. 1), to determine whether spontaneous mutations had occurred after the sample had been plated out (thus adding to the number of resistant colonies observed) and were distorting the results (Fig. 1). Macroconidia which generated visible colonies $4 \mathrm{~d}$ after inoculation were probably present in the original sample 


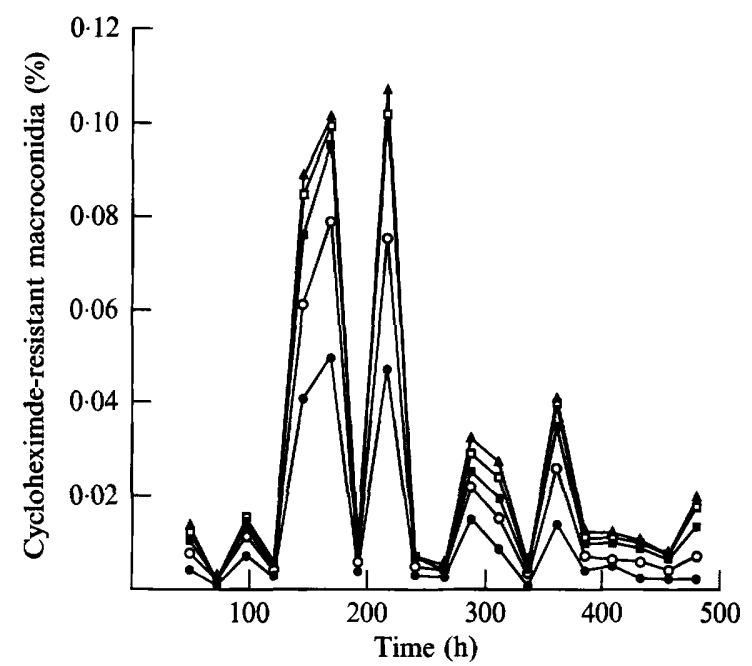

Fig. 1. Fluctuations in the proportion of cycloheximide-resistant macroconidia of $F$. graminearum during growth in glucose-limited chemostat culture $\left(25^{\circ} \mathrm{C}\right.$, pH $\left.5.8 \pm 0.1\right)$ at a dilution rate of $0.19 \mathrm{~h}^{-1}$ (experiment 1, Table 1). Cycloheximide-resistant colonies were counted after $4(\boldsymbol{O}), 5(\bigcirc), 6(\boldsymbol{\square}), 7(\square)$ and $8(\boldsymbol{\Delta}) \mathrm{d}$ and are expressed as a percentage of the total.

Table 1. Periods between peaks in the proportion of cycloheximide-resistant macroconidia in a population of F. graminearum

$F$. graminearum was grown in glucose-limited $[3 \mathrm{~g}$ glucose (l medium $)^{-1}$ ] chemostat cultures on modified Vogel's medium $\left(25^{\circ} \mathrm{C}, \mathrm{pH} 5.8 \pm 0.1\right)$ at a dilution rate of $0.19 \mathrm{~h}^{-1}$ (experiments $1-5$ ) or $0.06 \mathrm{~h}^{-1}$ (experiment 6). Samples were removed at regular intervals and the proportion of cycloheximide $(0.25 \mathrm{mM})$ resistant macroconidia in the macroconidial population was determined. In experiments 3 and 4 the proportion of cycloheximide-resistant macroconidia was still increasing when the experiments were terminated. The duration of each experiment is also indicated (total time).

\begin{tabular}{|c|c|c|c|c|c|c|c|}
\hline & \multicolumn{6}{|c|}{ Time between peak maxima (h) } & \multirow{2}{*}{$\begin{array}{l}\text { Total } \\
\text { time } \\
\text { (h) }\end{array}$} \\
\hline & $\begin{array}{c}\text { Peak } \\
0-1\end{array}$ & $\begin{array}{c}\text { Peak } \\
1-2\end{array}$ & $\begin{array}{c}\text { Peak } \\
2-3\end{array}$ & $\begin{array}{c}\text { Peak } \\
3-4\end{array}$ & $\begin{array}{c}\text { Peak } \\
4-5\end{array}$ & $\begin{array}{c}\text { Peak } \\
5-6\end{array}$ & \\
\hline Experiment 1 & 168 & 48 & 71 & 73 & & & 480 \\
\hline Experiment 2 & 171 & 168 & 96 & & & & 459 \\
\hline Experiment 3 & 120 & 144 & 120 & 168 & 96 & $>96$ & 744 \\
\hline Experiment 4 & 146 & 239 & 121 & $>119$ & & & 625 \\
\hline Experiment 5 & 72 & 192 & 96 & 96 & 168 & & 647 \\
\hline Experiment 6 & 78 & 120 & 192 & & & & 414 \\
\hline
\end{tabular}

when it was removed from the fermenter vessel, but the colonies present on the plates after $8 \mathrm{~d}$ incubation might have represented a mixture of spontaneous mutations which occurred in the fermenter and mutations which occurred subsequently on the plate. However, Fig. 1 illustrates that the day on which cycloheximide-resistant colonies were counted affected only the height of the adaptive peaks (presumably the result of a variable lag phase), not the time of their appearance. Further, there was very little increase in the number of cycloheximideresistant colonies after day 6 . In all subsequent experiments, cycloheximide-resistant colonies were counted on day 6 , day 7 or day 8 throughout the duration of a particular experiment.

\section{The occurrence of periodic selection}

Cycloheximide resistance was monitored in five glucoselimited chemostat populations of $F$. graminearum grown at a dilution rate of $0.19 \mathrm{~h}^{-1}$ and these populations were monitored for 459-744 h (equivalent to 119-202 generations, Table 1). In addition, a population grown at a dilution rate of $0.06 \mathrm{~h}^{-1}$ in a glucose-limited chemostat culture (experiment 6) was monitored for $414 \mathrm{~h}$ (36 generations). In all experiments, increases in the cycloheximide-resistant population were observed, followed by rapid decreases in the resistant population and subsequent increases, i.e. periodic selection was observed. These were similar to the pattern shown in Fig. 1 for experiment 1 . Highly branched, 'colonial' mutants were observed in only two experiments (nos. 3 and 4).

Three peaks of cycloheximide resistance were observed in Experiments 2 and 6, four in experiment 1 and 4, five in experiment 5 , and six in experiment 3 . The periods between respective peaks in each experiment are given in Table 1; the final peak in each experiment has been included in this Table, although the maximum peak height may not actually have been attained when the experiment was terminated. An average interval of $124 \pm 12 \mathrm{~h}$ (mean $\pm \mathrm{SE}$; the mean represents about 34 generations) was observed between consecutive peaks and $125 \pm 15 \mathrm{~h}$ between the consecutive troughs. The interval between peaks (or troughs) did not increase or decrease in a regular manner during the course of the experiments, and could be considered to be random.

In Experiments 3 and 4, highly branched, 'colonial' mutants were first observed at $648 \mathrm{~h}$ and $360 \mathrm{~h}$ (during the fifth and second peaks) respectively. No cycloheximide-resistant, highly branched, 'colonial' mutants were observed in experiment 3. In experiment 4, cycloheximide-resistant, 'colonial' mutants were observed after $554 \mathrm{~h}$ and accounted for the subsequent increase in the cycloheximide-resistant population (peak 4).

\section{Highly branched 'colonial' mutants}

The highly branched 'colonial' mutants observed in experiments 3 and 4 supplanted the more sparsely branched mycelial population. Selection coefficients for 


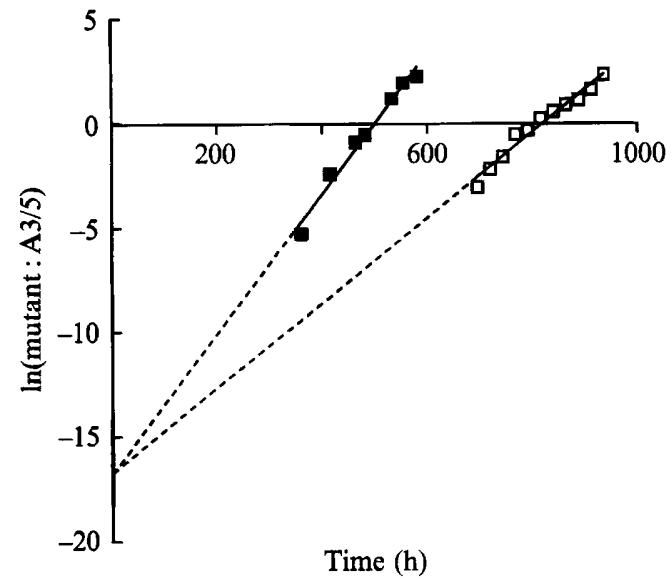

Fig. 2. Ratio of 'colonial' mutant to parent A3/5 (expressed as a natural logarithm) at different times after onset of continuous flow in the fermenter populations of experiments $3(\square)$ and $4(\square)$. Selection coefficients for the 'colonial' mutants of $0.020 \mathrm{~h}^{-1}$ (experiment 3 ) and $0.034 \mathrm{~h}^{-1}$ (experiment 4) were determined from the slope of the regression lines. The regression lines were extrapolated to $t=0 \mathrm{~h}$ (dashed lines); the point at which they crossed the $y$ axis provides an estimate of the concentration of mutant propagules in the populations at the start of continuous culture.

these colonial mutants were determined using the equation of Dykhuizen \& Hartl (1981):

$$
\ln \left[\frac{p(t)}{q(t)}\right]=\ln \left[\frac{p(0)}{q(0)}\right]+s t
$$

where $p(t)=$ concentration of one strain at time $t ; q(t)=$ concentration of the second strain at time $t ; p(0)$ and $q(0)=$ the initial concentrations of each strain; and $s=$ the selection coefficient. The selection coefficients for the 'colonial' mutants were $0.020 \mathrm{~h}^{-1}$ and $0.034 \mathrm{~h}^{-1}$ for experiments 3 and 4 respectively (Fig. 2).

Extrapolation of the regression lines used to determine the selection coefficients to the time when continuous medium flow was started ( $t=0$, Fig. 2), suggests that 'colonial' mutant propagules were already present in the population at the start of the continuous fermentation. The results (Fig. 2) suggest that approximately $5 \times 10^{-6} \%$ of the population consisted of 'colonial' mutant propagules. In a 2 litre fermenter there are at least $8 \times 10^{9}$ propagules (fragments of macroconidia) at $t=0$, and thus there were about 400 'colonial' mutant propagules present at the start of the continuous fermentations shown in Fig. 2.

\section{Monitoring periodic selection in second-generation macroconidia}

Second-generation macroconidia produced from samples from experiment 1 (samples 1-14, 48-360 h) were inoculated onto two concentrations of cycloheximide $(0.25$ and $0.75 \mathrm{~mm})$ and the number of resistant colonies

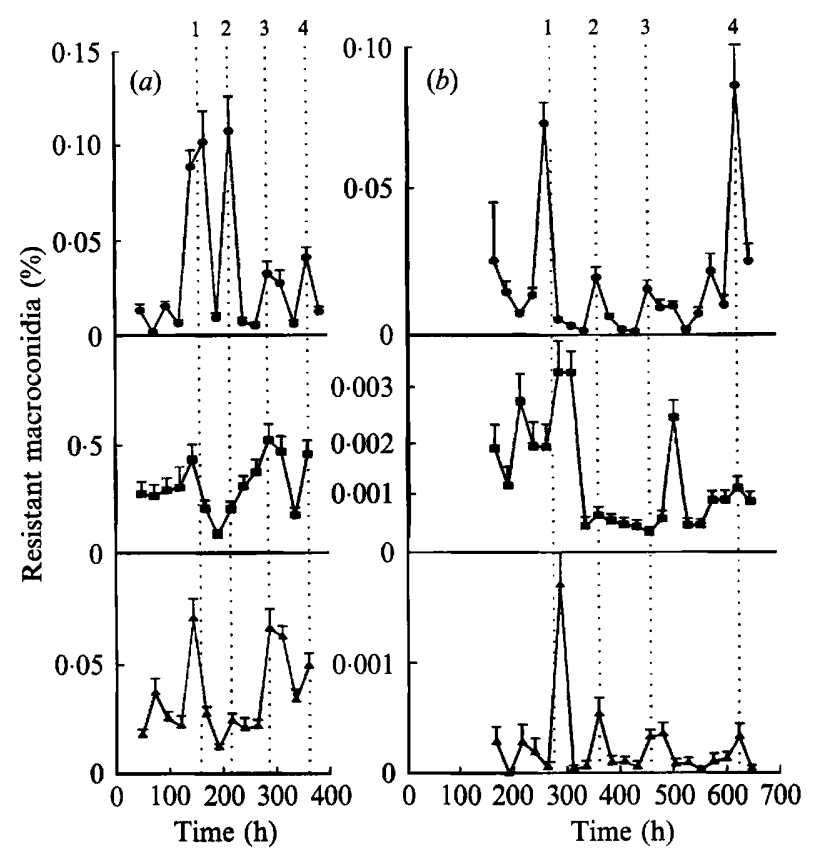

Fig. 3. Fluctuations in the proportion of resistant macroconidia in populations of $F$. graminearum grown in glucose-limited chemostat culture $\left(25^{\circ} \mathrm{C}\right.$, pH $\left.5 \cdot 8 \pm 0 \cdot 1\right)$ at a dilution rate of $0 \cdot 19 \mathrm{~h}^{-1}$, expressed as a percentage of total macroconidia. (a) Resistant populations in experiment 1 (Table 1; 0 , first-generation macroconidia grown on medium containing 0.25 mM-cycloheximide; $\square$, second-generation macroconidia grown on medium containing 0.25 mM-cycloheximide; $\boldsymbol{\Delta}$, second-generation macroconidia grown on medium containing 0.75 mM-cycloheximide). (b) Resistant populations in experiment 5 (Table 1; first-generation macroconidia grown on medium containing $\mathbf{0 . 2 5}$ mM-cycloheximide; $\square$, second-generation macroconidia grown on medium containing $300 \mathrm{~mm}$-potassium chlorate; $\boldsymbol{\Delta}$, secondgeneration macroconidia grown on medium containing $5.5 \mathrm{mM}-\mathrm{FPA}$ ). First-generation macroconidia were harvested directly from the chemostat population; second-generation macroconidia were harvested from plates mass-inoculated with first-generation macroconidia. The dotted lines indicate the peaks observed from first-generation macroconidia (with peak numbers indicated above). Note that the first peak in Table 1, experiment 5 is not indicated, as the first five samples were omitted in this experiment. Peak numbers refer to those shown in this graph. Error bars represent one standard error.

counted after $7 \mathrm{~d}$ incubation (Fig. $3 a$ ). With the exception of one point (at $216 \mathrm{~h}$, peak 2), the peaks and troughs observed in the first-generation macroconidia were also observed in second-generation macroconidia. An increase in the level of resistance (approximately 10fold) was observed in the second-generation macroconidia inoculated onto $0.25 \mathrm{mM}$-cycloheximide, compared with the first generation.

In a separate experiment, second-generation macroconidia from experiment 5 (samples 6-26, 168-647 h) were inoculated onto medium containing either potassium chlorate or FPA. The changes in the proportion of resistant colonies were compared with the cycloheximide-resistant population of the first generation (Fig. $3 b$ ). Generally, the peaks and troughs of the first- 
generation macroconidia were also observed in the second-generation macroconidia. However, the peaks observed in the second-generation macroconidia did not always correspond directly to those observed in the firstgeneration macroconidia. Both the FPA-resistant and the chlorate-resistant populations indicated that the first 'adaptive' peak occurred $24 \mathrm{~h}$ later than indicated by monitoring the cycloheximide-resistant population in the first generation. A similar shift of the third adaptive peak was observed in the chlorate-resistant population, but not in the FPA-resistant population. The second adaptive peak was not observed in the chlorate-resistant population.

\section{Discussion}

By monitoring cycloheximide resistance during prolonged continuous culture of $F$. graminearum it was possible to demonstrate that periodic selection of advantageous mutants occurred (Fig. 1, Table 1). During the first 450-744 h (118-202 generations) a change in the population adaptation occurred approximately every $124 \mathrm{~h}$ (about 34 generations). This was shorter than the intervals between adaptive peaks observed in bacterial populations (about $300 \mathrm{~h}$ or 86 generations: Helling $e t$ al., 1987; Koch, 1974), but similar to those observed in S. cerevisiae populations (about 145 h or 41 generations: Paquin \& Adams, 1983a). In the longest fermentation (experiment 3,744 h), the appearance of six peaks in the cycloheximide-resistant population suggested an accumulation of five selectively advantageous mutations. Cycloheximide-resistant 'colonial' mutants were not observed in this experiment, although non-resistant, 'colonial' mutants appeared in the population at $648 \mathrm{~h}$, indicating that the parental population had undergone changes prior to the appearance of 'colonial' mutations. Similarly, in experiment $4(625 \mathrm{~h})$ there appeared to be an accumulation of four mutations in the population, with the fourth presumably being the appearance of the highly-branched 'colonial' mutant. Thus, although the appearance of a 'colonial' mutant marked an adaptive change in the population, it was not the first adaptive change to occur in any of the populations studied.

The occurrence of adaptive peaks prior to the appearance of 'colonial' mutants implies that these morphological strains may contain multiple mutations. However, Dykhuizen \& Hartl (1981) noted that adaptation within the population, which occurred during the first $200 \mathrm{~h}$ (about 80 generations) of chemostatgrown Escherichia coli probably resulted from the selection of genetic variants, which were actually present in the inoculum. Adams \& Oeller (1986) noted that a similar situation probably occurred in Saccharomyces cerevisiae fermentations. They further suggested that the sequence of selection of advantageous (compared with the parental) strains present in the inoculum would depend not only on their relative selective advantages, but also on their initial concentrations in the population and on their interactions with each other. Adams \& Oeller's (1986) hypothesis is supported in the $F$. graminearum cultures by the evidence that 'colonial' mutant propagules were present at the start of the continuous fermentation in those experiments in which 'colonial' mutants were observed (Fig. 2). Presumably mutants which appeared prior to the 'colonial' mutants were also present during the batch fermentation and it is reasonable to assume that these contain only single mutations and that the order of their appearance was dependent on their relative concentrations at $t=0$, as well as on their selective advantage. The selective advantage of these early mutants does appear to have been very small, however; when strains were isolated from $339 \mathrm{~h}$ or $435 \mathrm{~h}$ of experiment 2 and placed in competition with the parental strain, A3/5, no measurable difference was found (M. G. Wiebe, unpublished results). Mutants arising in the population after the 'colonial' mutants have replaced most of the original population would be more likely to reflect mutations occurring after the onset of continuous operation.

Adaptive mutants were observed at the low dilution rate at the same frequencies as at the high dilution rate (Table 1). Dykhuizen \& Hartl (1981) also observed that $E$. coli evolved at the same rate at low $\left(0 \cdot 14 \mathrm{~h}^{-1}\right)$ and high $\left(0.28 \mathrm{~h}^{-1}\right)$ dilution rates. If mutants were present in the inoculum or arose during batch culture preceding chemostat culture, those mutants which had a selective advantage for growth at low dilution rates (e.g. mutants with lower saturation constants, $K_{\mathrm{s}}$, for the limiting substrate than the parent) would increase in the population at rates dependent on their initial concentration and selection coefficient (selective advantage). Alternatively, mutation rates may have been higher at low dilution rates than at high dilution rates. However, increased sporulation and fermentation at low dilution rates (Wiebe \& Trinci, 1991) might lead to an apparent increased mutation rate, because recessive mutants would be more rapidly isolated from densely sporulating and highly-fragmented cultures than from sparsely sporulating, less fragmented cultures.

The occurrence of periodic selection in fungal populations during long-term cultivation is a further indication of the heterogeneity of such populations. By monitoring periodic selection in populations of $F$. graminearum it became clear that there was considerable heterogeneity present early in the fermentation $(<72 \mathrm{~h}$, experiment 5 , Table 1 ). Much of this heterogeneity may have been present in the inoculum or may have resulted from an accumulation of mutations during the batch 
growth phase, prior to the onset of continuous cultivation. Therefore, it is obvious that inoculum preparation for either industrial or research fermentations should be as uniform as possible to reduce the degree of heterogeneity arising from this source. Mutations arising during batch cultivation, however, cannot be avoided.

The data in Fig. 3 showed that it was possible to monitor periodic selection in populations of filamentous fungi using second-generation macroconidia (macroconidia harvested from plates mass-inoculated with macroconidia taken directly from the chemostat). Unlike $F$. graminearum, many other fungi do not sporulate in continuous-flow cultures, or sporulate only at very low dilution rates. In the absence of spores, it would be difficult to monitor periodic selection in such populations directly from the fermenter culture, as use of mycelial fragments would result in dominant markers being overestimated, and recessive markers being underestimated. However, the use of second-generation spores to study periodic selection in such fungi would only be suitable for species with homokaryotic spores.

Fig. 3 also showed that second-generation macroconidia did not exactly mirror the adaptive peaks observed in the first generation. Adaptive peaks which were small in the first generation tended to become smaller in the second generation (see Fig. $3 b$, experiment 5 , peak 2, chlorate resistance). This occurred when the proportion of propagules bearing the mutation in the first generation of macroconidia was too low to be included in the sample used to inoculate the plate cultures from which second-generation macroconidia were eventually produced. Similarly, for some samples between peaks, mutant macroconidia might not be observed at all, as a result of the low initial concentration. Conversely, large peaks may occur in the second generation as a result of their chance over-representation in the sample of macroconidia used to inoculate the plates.

Recessive markers for chlorate and FPA resistance sometimes indicated a later appearance of the peak than was indicated by the dominant cycloheximide resistance marker in the first-generation macroconidia. This may be the result of random selection in the subculture, as described above, or it may indicate that the actual peak fell somewhere between those indicated by the different markers. More frequent sampling could be used to determine the timing of each peak precisely. It was concluded that both chlorate and FPA resistance, as well as cycloheximide resistance, were useful genetic markers for monitoring periodic selection in cultures of $F$. graminearum.

Koch (1974) noted that periodic selection tended to limit the development of genetic heterogeneity in a population. Thus, in a continuous culture, the increase in population heterogeneity with time would be limited by the periodic displacement of the increasingly heterogeneous population by a better adapted strain which is likely to resemble the parental strain quite closely. However, some fed-batch and repeated fed-batch cultures may be maintained for up to $7 \mathrm{~d}(168 \mathrm{~h})$ or longer. In these culture systems, a continual increase in culture heterogeneity would be expected, either because none of the population is being removed (fed-batch), or because only a part of the population is removed after long time intervals (repeated fed-batch). In addition, selective pressures favouring low growth rates may act on this heterogeneity (see Table 1 , experiment $6, D=0.06 \mathrm{~h}^{-1}$ ), resulting in a shift in the proportion of the population which retains the initial phenotype of the culture. Such a shift would be comparable to the 'jackpot' effect observed in batch cultures (Kubitschek, 1970). An increase in the proportion of non-secreting mutants of Aspergillus oryzae, for example, may account for the decline in protein secretion which has been observed at the end of fed-batch cultures of this species (K. Hansen, personal communication).

We thank the Science \& Engineering Research Council and Marlow Foods for supporting this work.

\section{References}

ADAMS, J. \& OELLER, P. W. (1986). Structure of evolving populations of Saccharomyces cerevisiae: adaptive changes are frequently associated with sequence alterations involving mobile elements belonging to the Ty family. Proceedings of the National Academy of Sciences of the United States of America 83, 7124-7127.

CharlesworTh, B. (1983). Adaptive evolution in the laboratory. Nature, London 302, 479-480.

DyKhUIZEN, D. \& HARTL, D. (1981). Evolution of competitive ability in Escherichia coli. Evolution 35, 581-594.

DykhuIzen, D. E. \& HARTL, D. L. (1983). Selection in chemostats. Microbiology Reviews 47, 150-168.

Helling, R. B., Vargas, C. N. \& Adams, J. (1987). Evolution of Escherichia coli during growth in a constant environment. Genetics 116, 349-358.

Hsu, K. S. (1963). The genetic basis of actidione resistance in Neurospora. Journal of General Microbiology 32, 341-347.

KoCH, A. L. (1974). The pertinence of the periodic selection phenomenon to prokaryote evolution. Genetics 77, 127-142.

KUBITSCHEK, H. E. (1970). Introduction to Research with Continuous Cultures. Englewood Cliffs, NJ: Prentice-Hall.

Miller, J. J. (1946). Cultural and taxonomic studies on certain Fusaria. I. Mutations in culture. Canadian Journal of Research 24 (C), 188-212.

Novick, A. \& SzILARD, L. (1950). Experiments with the chemostat on spontaneous mutation of bacteria. Proceedings of the National Academy of Sciences of the United States of America 36, 708-719.

PAQUIN, C. \& ADAMs, J. (1983a). Frequency of fixation of adaptive mutations is higher in evolving diploid than haploid yeast populations. Nature, London 302, 495-500.

Paquin, C. \& Adams, J. (1983b). Relative fitness can decrease in evolving asexual populations of Saccharomyces cerevisiae. Nature, London 306, 368-371.

Pongratz, M. \& KlingmülleR, W. (1973). Role of ribosomes in cycloheximide resistance of Neurospora mutants. Molecular and General Genetics 124, 359-363. 
Trincr, A. P. J. \& GULL, K. (1970). Effect of actidione, griseofulvin and triphenyltin acetate on the kinetics of fungal growth. Journal of General Microbiology 60, 287-292.

VoGEL, H. J. (1956). A convenient growth medium for Neurospora (Medium N). Microbial Genetics Bulletin 13, 42-44.

WIEBE, M. G. \& TrINCI, A. P. J. (1991). Dilution rate as a determinant of mycelial morphology in continuous culture. Biotechnology and Bioengineering 38, 75-81.

Wiebe, M. G., Trinci, A. P. J., Cunliffe, B., Robson, G. D. \& Oliver, S. G. (1991). Appearance of morphological (colonial) mutants in glucose-limited, continuous flow cultures of Fusarium graminearum A3/5. Mycological Research 95, 1284-1288. 